Fecha de recepción: 30 de septiembre de 2020 Fecha de evaluación: 27 de noviembre de 2020 Fecha de aprobación: 16 de diciembre de 2020

\section{Espacios de encierro dentro del encierro. Análisis de un centro cerrado para jóvenes procesados penalmente en Argentina*}

\section{Federico Eduardo Ortubey ${ }^{* \star}$}

\section{Para citar este artículo}

Urtubey, F. (2021). Espacios de encierro dentro del encierro. Análisis de un centro cerrado para jóvenes procesados penalmente en Argentina. Vía luris, 30, 55-71. https://doi.org/10.37511/viaiuris.n30a2

\title{
RESUMEN
}

El análisis de los espacios de encierro constituye un ámbito de indagaciones interesante para conocer y estudiar las prácticas de gobierno que se despliegan en los contextos carcelarios. La delimitación de espacios de privilegio, de uso común o bien de mayor imposición de tormentos, desplegada en función de los mecanismos de gobierno sobre los internos, son factores que organizan una geografía carcelaria, moldeada mediante usos y prácticas sociales que solo pueden ser identificados mediante la observación in situ. Este artículo propone el análisis de un centro cerrado de privación de la libertad para jóvenes procesados penalmente en la ciudad de La Plata, provincia de Buenos Aires (Argentina). El objetivo general está dado por indagar de qué manera la dimensión espacial y arquitectónica del centro cerrado constituye una dimensión expresiva de la racionalidad punitiva del dispositivo penal. Se utilizó una metodología de corte cualitativo, tomando como referencia fuentes primarias (notas de campo y entrevistas) producto de un trabajo de campo de duración bianual. Algunos resultados del estudio dan luz sobre las intersecciones entre diversas prácticas de poder que moldean el gobierno de los sujetos en el encierro penal, y permiten identificar especialmente el lugar que ocupa el funcionamiento de la organización espacial en estas dinámicas.

* El presente trabajo se enmarca en el proyecto posdoctoral del autor, titulado "Discursos judiciales en torno a jóvenes en conflicto con la ley penal. Análisis de causas del Fuero de Responsabilidad Penal Juvenil del Dpto. Judicial La Plata" (FaHCE, UNLP), bajo la dirección de la Dra. Angela Oyhandy. Periodo. 2020-2022. La plata, Argentina.

** Investigador PosDoctoral, Doctor y Magister en Ciencias Sociales en la Facultad de Humanidades y Ciencias de la Educación de la Universidad Nacional de La Plata - UNLP. Abogado (Facultad de Ciencias Jurídicas y Sociales, Universidad Nacional de La Plata). Docente de las cátedras Derecho 1 y Derecho 2. La plata, Argentina. Correo electrónico de contacto: ue.federico@gmail.com. Orcid: http://orcid.org/0000-0003-1392-159X.
DOI: https://doi.org/10.37511/viaiuris.n30a2

Creative Commons Atribución-NoComercial-Compartirlgual 4.0 Internacional (CC BY-NC-SA 4.0).

\section{(c) (1) (8)}

\section{Palabras clave}

Justicia penal juvenil, prisiones, jóvenes, sociología penal, dispositivo. 


\title{
Confinement spaces within the confinement. Analysis of a High Security Center for prosecuted youths in Argentina
}

\author{
Federico Eduardo Ortubey
}

\begin{abstract}
The analysis of confinement spaces presents itself as an interesting field of investigation to study the governing practices put on display in prisons. The delimitation of spaces of privilege, of common use or either of greater imposition of punishment, which are deployed based on the governing mechanisms that are put into work over the inmates, are organizing factors of a prison geography, shaped by social customs and practices that can only be identified through in situ observation. This paper aims to explore and describe a High Security Center for criminal youths, in the city of La Plata, state of Buenos Aires, Argentina. Its main goal is to inquire into the way in which the spatial and architectural dimension of the High Security Center constitutes an expressive dimension of the punitive rationality of the penal device. In order to do that, a qualitative methodology has been used, taking as reference primary sources such as field notes and interviews, which are the product of a two years field work. Some of the results of this research shed light over the intersections between different power practices that shape the governing of subjects in confinement, allowing to specially identify the place occupied by the functioning of a spatial organization within those dynamics.
\end{abstract}

\section{Keywords}

Criminal justice system, prisons, juveniles, youths, criminal sociology, devices. 


\title{
Espaços de confinamento dentro do confinamento. Análise de um centro fechado para jovens em processo penal na Argentina
}

\author{
Federico Eduardo Ortubey
}

\section{RESUMO}

A análise dos espaços de confinamento é um campo de investigação interessante para conhecer e estudar as práticas governamentais que são implantadas em contextos prisionais. A delimitação de espaços de privilégio, de uso comum ou de maior imposição de tormentos, implantados em função dos mecanismos do governo sobre os detentos, são fatores que organizam uma geografia carcerária, moldada por meio de usos e práticas sociais que só podem ser identificados por meio da observação in situ. Este artigo propõe a análise de um centro fechado de privação de liberdade para jovens criminalmente processados na cidade de La Plata, província de Buenos Aires, Argentina. O objetivo geral é investigar de que forma a dimensão espacial e arquitetônica do centro fechado constitui uma dimensão expressiva da racionalidade punitiva do dispositivo penal. Foi utilizada uma metodologia qualitativa, tomando como referência fontes primárias (notas de campo e entrevistas) produto de um trabalho de campo bianual. Alguns resultados do estudo lançam luz sobre as interseções entre várias práticas de poder que moldam o governo dos sujeitos no confinamento penal, permitindo identificar especialmente o lugar do funcionamento da organização espacial nestas dinâmicas.

Palavras-chave

Justiça penal juvenil; prisões; juventude; sociologia penal; disposição. 


\section{Des espaces de confinement dans le confinement. Analyse d'un centre fermé pour mineurs faisant l'objet de poursuites pénales en Argentine}

\section{Federico Eduardo Ortubey}

\section{RÉSUMÉ}

L'analyse des espaces d'enfermement est un champ d'investigation intéressant pour connaître et étudier les pratiques gouvernementales qui se déploient dans les contextes carcéraux. La délimitation d'espaces de privilège, d'usage commun ou de plus grande imposition de supplices, déployés en fonction des mécanismes de gouvernement sur les détenus, sont des facteurs qui organisent une géographie carcérale, modelée au moyen d'usages et de pratiques sociales qui ne peuvent être identifiés qu'au moyen de l'observation in situ. Cet article propose l'analyse d'un Centre Fermé de privation de liberté pour les jeunes poursuivis pénalement dans la ville de La Plata, province de Buenos Aires, Argentine. L'objectif général est d'étudier en quoi la dimension spatiale et architecturale du Centre Fermé constitue une dimension expressive de la rationalité punitive du dispositif pénal. Une méthodologie qualitative a été utilisée, prenant comme référence des sources primaires (notes de terrain et entretiens) issues d'un travail de terrain biannuel. Certains résultats de l'étude mettent en lumière les intersections entre les différentes pratiques de pouvoir qui façonnent le gouvernement des sujets dans l'enfermement pénal, permettant notamment d'identifier la place du fonctionnement de l'organisation spatiale dans ces dynamiques.

Mots-clés

Justice pénale juvénile; prisons; jeunesse; sociologie pénale; dispositif. 


\section{INTRODUCCIÓN}

Este artículo propone analizar las características de un espacio constructivo concreto destinado a la privación de la libertad de jóvenes procesados penalmente en la ciudad de La Plata, provincia de Buenos Aires (Argentina), con el objetivo de indagar de qué manera su forma arquitectónica influye en la producción del orden carcelario y el gobierno de la población intra muros. En la mencionada localidad existe un vasto conjunto de dispositivos penales de diversa naturaleza, respecto de los cuales investigaciones como las de Daroqui, López y Cipriano (2012), Guemureman (2015) y López (2010; 2012) han dado cuenta de las modalidades de sujeción y coerción que se disponen sobre las personas allí alojadas. Prosiguiendo estas líneas de análisis que buscan dar luces sobre las condiciones de vida de las personas en situación de encierro punitivo, el presente artículo se apoya en un trabajo de campo realizado entre 2018 y 2019, el cual permitió una aproximación sistemática y continuada a la vida cotidiana de los jóvenes alojados en un establecimiento cerrado del sistema penal juvenil bonaerense ubicado en las afueras de la localidad de La Plata. Así, se realizará una indagación cualitativa en torno a un estudio de caso en particular, ya que se propone describir y analizar de qué manera el "despliegue real del castigo" (Daroqui et al., 2012) también es producido a partir de una forma arquitectónica que facilita una gestión de su "clientela"1 penal, en un espacio de detención concreto.

Como necesaria introducción al tema, cabe contextualizar que, en la provincia de Buenos Aires, las personas jóvenes menores de 18 años procesadas penalmente y sobre las cuales se dicta una medida de seguridad privativa de la libertad son competencia del Sistema de Responsabilidad Penal Juvenil provincial. Este sistema no está creado por una norma en particular, sino que se monta sobre diversas disposiciones, entre ellas la Ley Provincial 13.634 de 2006. Teniendo en cuenta que la República Argentina adoptó la forma de Estado federal

1 La idea de "clientela" pretende dar cuenta de la selectividad del sistema penal, en la medida en que son determinadas capas sociales las que mayoritariamente se constituyen como objeto de persecución penal, políticas de encarcelamiento y otras estrategias complementarias de gobierno. Desde la perspectiva criminológica, podría señalarse que el concepto se vincula a las teorías del etiquetamiento (labeling approach), que enfatizan cómo se despliegan los procesos de criminalización. en el artículo 1 de la Constitución Nacional, es preciso señalar que las leyes penales son sancionadas por el Congreso Nacional -según lo normado en el artículo 75 inciso 12 del texto constitucional-, y tienen validez en todo el territorio de la Nación, en tanto la regulación de los procesos es incumbencia de cada provincia o estado miembro. Así, en cuanto al sistema penal y penal juvenil, las normas de validez nacional son el Código Penal y el Decreto-Ley 22.278 o Régimen Penal de la Minoridad, respectivamente. En cuanto al orden local, en la jurisdicción de la provincia de Buenos Aires es preciso dar cuenta de la Ley 13.298 -que despliega el denominado Sistema de Protección y Promoción de Derechos, el cual consagra diversos derechos y obligaciones del Estado y la sociedad civil en general respecto de los niños, niñas y adolescentes, sin distinción alguna- y fundamentalmente de la mencionada Ley 13.634, que restructuró el proceso de familia y el proceso penal juvenil. Esta última norma constituye el armazón legal que estructura el sistema penal juvenil provincial -donde se inserta nuestro estudio de caso- $y$ establece lineamientos a los que deben adecuarse los espacios destinados al encierro punitivo de jóvenes.

En cuanto a los dispositivos de detención de jóvenes, la Resolución Provincial 172 de 2007 y sus modificatorias tipifican los diversos espacios donde se adelanta el cumplimiento de las medidas restrictivas de la libertad ${ }^{2}$. Esta tipología comprende centros de referencia, recepción, contención y centros cerrados, y sobrevuela en esta creación normativa el principio de la prevención especial positiva, esto es, de que la privación de la libertad puede ser concebida como parte de una intervención reparadora sobre los sujetos, tal como señala López (2012) en una investigación sobre el tema. Ahora, si bien el encierro se presenta como una medida socioeducativa, cierto es que la norma mencionada no conceptualiza el sentido de esta (Fasciolo, 2015), lo que guarda correlación con la circunstancia de que "no existen materiales oficiales formales y estructurados de formación, capacitación o difusión a nivel ministerial [...] solo en algunos pocos proyectos

2 La Ley 13.634 establece la finalidad educativa y responsabilizante del proceso penal, y en el caso de que se dicten autos judiciales que establezcan la restricción de la libertad de un joven procesado, se anuncia -por ejemplo en el artículo 81 de dicha ley- que estas serán cumplidas en espacios en los que obligatoriamente se ofrecerán "actividades socio-pedagógicas" a los jóvenes. 
institucionales [...] se exhibe alguna definición sobre sus objetivos y fundamentación de la intervención" (Daroqui et al., 2012, p. 257).

En el marco descripto, la perspectiva de este artículo se posiciona desde el campo de la sociología y del subcampo de la sociología penal juvenil, para indagar la materialidad de los espacios de encierro. El estado del arte involucra el campo de estudios de la sociología penal juvenil y el de las políticas de seguridad en la Argentina contemporánea. En la primera década de este siglo, y fundamentalmente a partir de las leyes mencionadas, que se sancionaron y reglamentaron entre 2005 y 2007, la mayoría de las intervenciones teóricas tuvieron como eje los cambios instrumentados en la normativa local en torno al desarme del viejo modelo tutelar, que regulaba la infancia criminal, y la transición hacia un nuevo paradigma de protección y promoción integral de derechos de los niños, niñas y adolescentes, tendiente en lo más significativo a separar expresamente las competencias y causas penales de las asistenciales, tal como se profundiza en los trabajos de Villalta (2013), Beloff (2008) y Guemureman (2005), entre muchos otros. Habiendo transcurrido tal fase de reacomodamiento legislativo e institucional, durante la última década se destaca una gran profusión de aportes que abordan las violencias institucionales propias de cada eslabón de la cadena punitiva (en su engranaje policial, judicial y custodial) que se despliegan sobre los jóvenes aprehendidos. Entre ellos se destaca la investigación colectiva de Daroqui et al. (2012), así como otros estudios en torno a las políticas de seguridad dirigidas a la niñez y la adolescencia en el orden nacional y provincial, como en el caso de Guemureman (2015).

Por su lado, los informes realizados por la Comisión Provincial por la Memoria de la provincia de Buenos Aires -organismo público autónomo y autárquico-, cuya última edición se realizó en 2020, analizan desde un enfoque cuantitativo la situación de la población del sistema penal en general y del juvenil en particular. Su valioso aporte contrasta con la desidia de la producción estadística e informativa por parte de las agencias estatales encargadas del despliegue del sistema penal en general y del penal juvenil en particular, tal como se pone de manifiesto en el libro colectivo de Daroqui et al. (2012), el cual sistematiza un estudio multidimensional sobre el sistema penal juvenil provincial y las condiciones de encierro en los dispositivos punitivos $^{3}$.

Dicho lo anterior, este escrito se propone analizar de qué manera la forma arquitectónica de un centro de detención penal importa, en los términos de Foucault (2005), una dimensión que puede ponerse en diálogo con la lógica de gobierno interno institucional. En efecto, desde las ciencias sociales el centro cerrado puede ser entendido como una institución total (Goffman, 1961) o de secuestro (Foucault, 2005), donde impera una absoluta regimentación de la vida de los sujetos que involuntariamente la habitan, a partir de una jerarquía institucional que separa a los observadores y administradores del orden, de los observados y sujetos al encierro. El abordaje de las condiciones de detención de los jóvenes en general y de la dimensión de la administración del espacio en particular constituye un eje de importancia para determinar las prácticas segregativas y disciplinantes que, por detrás de lo anunciado en las disposiciones normativas, se dan en el nivel de las prácticas sociales. De tal modo, en las conclusiones se evidencia cómo la compartimentación de los espacios al interior de un establecimiento de encierro de jóvenes constituye una variable de análisis para conocer la modulación, administración y gestión del poder de castigar y construir del orden interno.

\section{METODOLOGÍA}

En cuanto a las precisiones metodológicas, en primer término corresponde destacar que este artículo parte de la reconstrucción de un estudio de caso, desde una perspectiva de corte cualitativo. Los estudios de caso suponen una estrategia de investigación dirigida a comprender las dinámicas presentes en contextos singulares (Eisenhardt, 1989) y pueden

3 Vinculado a este punto en particular, es menester señalar que el departamento de investigaciones de la Procuración de la Suprema Corte de Justicia es el único que hace una aproximación estadística a la situación de los jóvenes en el sistema penal en la provincia de Buenos Aires. La Procuración posee un Sistema Informático del Ministerio Público (SIMP) a partir del cual se elaboran informes institucionales anuales sobre la cantidad de causas penales iniciadas, dato que se desagrega en torno al departamento judicial donde estas tuvieron inicio, el bien jurídico afectado por el delito en cuestión, entre otras variables. Sin embargo, no existen documentos institucionales que den cuenta de las condiciones de encierro de la población juvenil -ni tampoco respecto del fuero de mayores- alojada en centros de detención, aspecto que solo se encuentra en los informes de la Comisión Provincial por la Memoria. 
admitir distintos métodos para la recolección de evidencia cualitativa o cuantitativa (Martínez, 2006).

Las referencias empíricas estuvieron dadas por un trabajo de campo realizado en el centro cerrado de las afueras de la ciudad de La Plata, durante un periodo de casi dos años que transcurrió entre febrero de 2018 y diciembre de 2019. Es sabido que el ingreso a las unidades penales tanto de jóvenes como de mayores de edad suele ser un escollo fundamental para los interesados en desarrollar investigaciones empíricas sobre las dinámicas carcelarias. Este impedimento fue superado en el presente estudio, en la medida en que el acceso al centro cerrado se produjo acompañando a un equipo de extensión universitaria conformado por cuatro agentes docentes y no docentes de la Facultad de Artes de la Universidad Nacional de La Plata, el cual contaba con los permisos administrativos respectivos para el ingreso a dicha unidad penal, y sus integrantes autorizaron al autor de este artículo a acompañarlos en cada encuentro. También se permitió llevar un diario de campo para registrar lo acontecido en cada jornada, cuyas notas se transcriben y analizan en este texto.

Cabe reconocer que la factibilidad del trabajo de campo se asentó principalmente en el vínculo institucional con el referido equipo extensionista. Al mismo tiempo, es muy importante dejar en claro que el hecho de acompañar y presenciar las experiencias educativas permitió establecer contacto tanto con los jóvenes privados de su libertad como con los operadores institucionales encargados de la administración del centro cerrado. Finalmente, es preciso destacar que la presencia en el espacio penal se dio sin que los extensionistas requirieran al autor de este artículo la realización de intervenciones activas en el devenir de las experiencias pedagógicas.

Durante el periodo en el cual se realizó el trabajo de campo se contabilizaron aproximadamente unas 45 visitas al centro cerrado, consignadas todas, con sus respectivos hallazgos y observaciones en general, en el mencionado diario de campo. Las técnicas desplegadas fueron observación participante y no participante, además de entrevistas estructuradas y semiestructuradas y abiertas a informantes clave -entre ellos 17 jóvenes detenidos y el personal de la institución-, sobre cuyo resultado se realizó un análisis de contenido (Scribano, 2008; Valles, 2000). Las observaciones permitieron conocer in situ las formas de habitar el espacio penal y los diversos procesos de autorización y desautorización involucrados en las posibilidades de los jóvenes de circular por diversos ambientes del establecimiento penal. Por su parte, las entrevistas posibilitaron una aproximación a las lógicas y los sentidos implicados en dichas oportunidades de circulación, así como conocer de qué manera este gerenciamiento del espacio penal condiciona las trayectorias de los jóvenes en el encierro.

Si bien en las páginas subsiguientes se analiza en profundidad la morfología del establecimiento de encierro, no está de más dejar en claro que el centro cerrado analizado -donde se ejecutan medidas de seguridad privativas de la libertad- presenta una capacidad de 25 plazas para jóvenes de sexo masculino, de los cuales el 70 \% tenían entre 16 y 18 años en el momento en que se realizó el trabajo de campo, y el $30 \%$ tenían entre 18 y 21 años $^{4}$. Parte de su funcionamiento se encuentra regulado por la Resolución Provincial 172 de 2007 y sus modificatorias, bajo la competencia de la Dirección de Institutos Penales, es decir, dentro del Organismo de Niñez y Adolescencia de la provincia, que a su vez está en la órbita del Ministerio de Desarrollo de la Comunidad. Puede afirmarse que el centro cerrado sería el equivalente a una institución de máxima seguridad.

\section{MARCO TEÓRICO}

Para conceptualizar el espacio y las mediaciones que se dan en él, es oportuno mencionar una de las observaciones que realiza Pierre Bourdieu en su libro La miseria del mundo (1999), en cuanto a que el espacio es claramente uno de los ámbitos donde se produce una afirmación y ejercicio del poder. El autor argumenta que esta condensación se produce

en la forma más sutil, la de la violencia simbólica como violencia inadvertida: los espacios

4 El mencionado Decreto-Ley 22.278 o Régimen Penal de la Minoridad estableció que no son punibles los menores de 16 años. Aunque en Argentina la mayoría de edad se adquiere de pleno derecho a los 18 años, si al cumplir esta edad un joven se encuentra privado de su libertad por imperio de una medida de seguridad dictada en el marco un proceso penal, aquel puede continuar su internación en un establecimiento penal juvenil, es decir, sin necesidad de ser trasladado a un establecimiento penal para mayores. Por esta razón se pudo interactuar con jóvenes mayores de 18 años en el Centro Cerrado analizado. 
arquitectónicos -cuyas conminaciones mudas interpelan directamente al cuerpo y obtienen de este, con tanta certeza como la etiqueta de las sociedades cortesanas, la reverencia, el respeto que nace de alejamiento o, mejor, del estar lejos, a distancia respetuosa- son en verdad los componentes más importantes, a causa de su misma invisibilidad, de la simbólica del poder y de los efectos totalmente reales del poder simbólico. (Bourdieu, 1999, p. 122)

La mirada del sociólogo francés permite pensar introductoriamente de qué manera tanto la materialidad de una institución como los rituales y comportamientos que la sostienen hacen parte de su efecto de subjetivación. Entretanto, Michel Foucault (2005) analiza el surgimiento y la expansión de la forma propiamente carcelaria, a la luz del proceso de abandono del espectáculo de la pena física y la publicidad de los tormentos, y la entrada en una época de sobriedad punitiva, en la que se persigue más bien que el castigo "actúe en profundidad sobre el corazón, el pensamiento, la voluntad, las disposiciones" (p. 24). Así, el autor argumenta que "el castigo tenderá, pues, a convertirse en la parte más oculta del proceso penal" (2005, p. 17) y será negado como tal, presentándose como una posibilidad de saneamiento y purificación de los condenados.

Es de resaltar que el mismo Foucault (2005) señala, considerando las distintas tecnologías que expresan el castigo penal intra muros, que al fin y al cabo este nunca dejó de ser, en algún punto, corporal: las distintas técnicas y rutinas impuestas sobre los cuerpos de los condenados dan cuenta de esta premisa, más allá de que su fundamento radique en una corrección de tipo espiritual o moral. Por su lado, aún el énfasis en torno al abordaje socioeducativo del encierro mencionado anteriormente auspicia pensar los espacios de encierro como instancias en las cuales es preciso intervenir en la conducta de los jóvenes alojados en el sistema penal. Los espacios precarios que constituyen las instituciones penales juveniles y el ideario correccional en el cual estas tienen asidero traduce nuevamente el concurso del "suplemento penitenciario" (Foucault, 2005), es decir, aquellas técnicas y tecnologías que se imprimen coercitivamente sobre los sujetos, con el fin de introyectar la terapéutica del castigo. Esta constatación evidencia que la configuración del poder disciplinario de las instituciones penales descansa y se organiza sobre una madeja compuesta por el andamiaje legal, la forma arquitectónica que entrelaza el encierro, los saberes y las técnicas disciplinantes específicas que organizan y dan cuerpo a distintas formas de despliegue de los espacios de la prisión.

En relación con lo dicho hasta aquí y en la medida en que el objetivo de este artículo es demostrar cómo que la forma arquitectónica constituye una dimensión en la que es posible identificar las marcas que orientan el despliegue de lo punitivo, es dable destacar que la gubernamentalidad intra muros genera un conjunto de dispositivos que en su intersección organizan una economía de los cuerpos en el encierro -en un sentido amplio y complejo del cuerpo que excede a su mera materialidad-. Así, dentro de un mismo espacio carcelario se producen y desdoblan una serie de espacios diferenciales ${ }^{5}$, en los cuales el rigor punitivo se expresa y gestiona de manera disímil. Así, estos espacios carcelarios organizan un archipiélago de lugares de cierto beneficio y otros lugares de mayor conminación. Desde esta lectura, en la siguiente sección interesa detectar, analizar e indagar estas dimensiones de despliegue de la imposición coercitiva en el espacio constructivo concreto del centro cerrado objeto del presente artículo.

\section{RESULTADOS Y DISCUSIÓN}

Los resultados se abordan en dos secciones fundamentales y diferenciadas. La primera se centra en el análisis de la forma arquitectónica del centro cerrado, describiendo en profundidad cómo esta presenta la existencia de ámbitos más cruentos y de mayor degradación para los internos, respecto de otros lugares de menor rigor. Se destaca que tal relación es expresiva de la modulación del castigo penal, y su reflejo en espacios y compartimentos diferenciados y reservados para momentos y ocasiones específicas de la vida de los jóvenes en la institución. En la segunda sección, los resultados del análisis permiten conocer de qué manera el espacio

5 Cuando se habla de "espacio diferencial" no se alude a la conceptualización homóloga realizada por Lefebvre (1991; 2008) para oponer estos a los "espacios abstractos" o "dominantes". Con "espacios diferenciales" se alude en este artículo a los espacios propiamente carcelarios donde concurren distintas expresiones del poder punitivo, tendientes a generar diversas formas de endurecimiento que, por su carácter relacional, redundan en espacios considerados "de beneficio" y otros más degradados y degradantes, caracterizados por una mayor aplicación de dolor. 
interno de la cárcel siempre está en tensión con una realidad extra muros que se percibe o anuncia como amenazante, y que en cualquier caso fomenta un gobierno del encierro con mayores niveles de discrecionalidad para limitar la comunicación de los jóvenes con personas externas a la institución, como sus familiares.

\section{El encierro dentro del encierro}

Desde los primeros instantes en que comenzó a realizarse el trabajo de campo en el centro cerrado, a principios de febrero de 2018, asomó con nitidez que el contexto de encierro distaba de ser una geografía homogénea y que, por el contrario, estaba constituido por diversas regiones en las cuales el poder disciplinario se expresaba de forma diferencial. De ello se tomó nota en los inicios del acompañamiento al proyecto de extensión de la Facultad de Artes, cuando comenzó una dinámica de encuentros quincenales con los jóvenes privados de su libertad que se extendería hasta diciembre de 2019. Este proyecto de extensión tenía prevista la realización de actividades artísticas y educativas que fueron llevadas a cabo en un despliegue no exento de tensiones, a causa de las pretensiones correctivas dispuestas desde el establecimiento penal.

De conformidad con lo expuesto hasta aquí, cuando se acompañó a las extensionistas al centro cerrado durante los primeros meses de 2018, se pudo constatar en forma algo inmediata una separación del contexto de encierro en dos grandes espacios. En este edificio de dos plantas pudo observarse la existencia de un gran espacio constituido por diversas instalaciones del centro cerrado propiamente dicho (las oficinas de los administradores del instituto, las "habitaciones" de los jóvenes, un salón de usos múltiples y un espacio destinado al desarrollo de actividades escolares) y un espacio de rezago ubicado a la izquierda de la planta superior del edificio. Este se denominaba "alcaldía" y se disponía como el primer espacio al que los jóvenes accedían en cumplimiento de la medida de privación de la libertad dispuesta sobre ellos. Como se irá recorriendo a lo largo del artículo, la alcaldía distaba de ser un mero espacio circunstancial de ingreso para los jóvenes; por el contrario, implicaba el primer eslabón de la etapa "custodial" de la cadena punitiva. Desde este punto de vista, el trato considerablemente más cruento que los jóvenes recibirían en este recinto implicaría a la postre el ingreso a la díada relacional y diferencial constituida por espacios "mejores" y "peores", y dentro de la cual la alcaldía se ubicaría como un punto de referencia central en la cartografía institucional.

Figura 1. Esquema de distribución de la planta inferior del centro cerrado

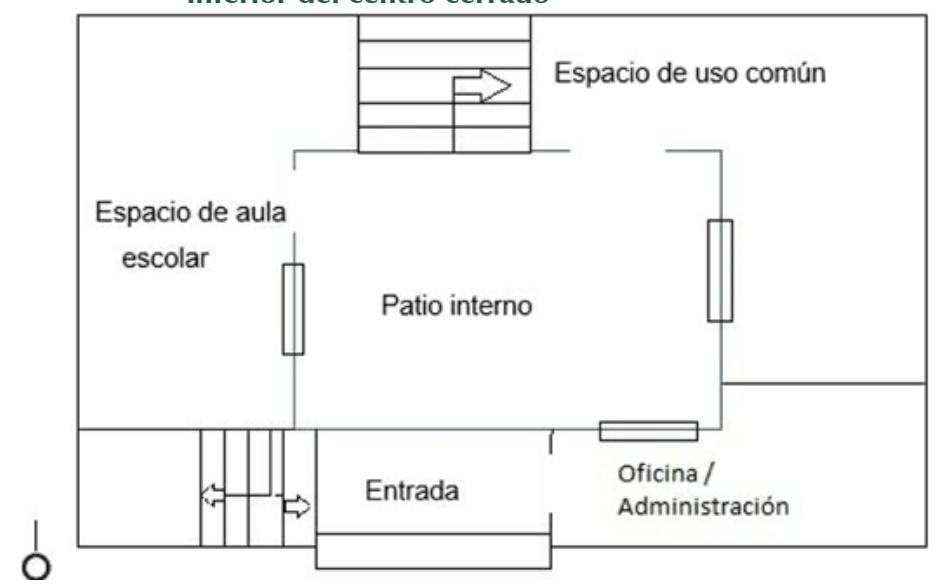

Fuente: elaboración propia.

Figura 2. Esquema de distribución del piso superior del centro cerrado (a la izquierda las celdas que componen la alcaldía)

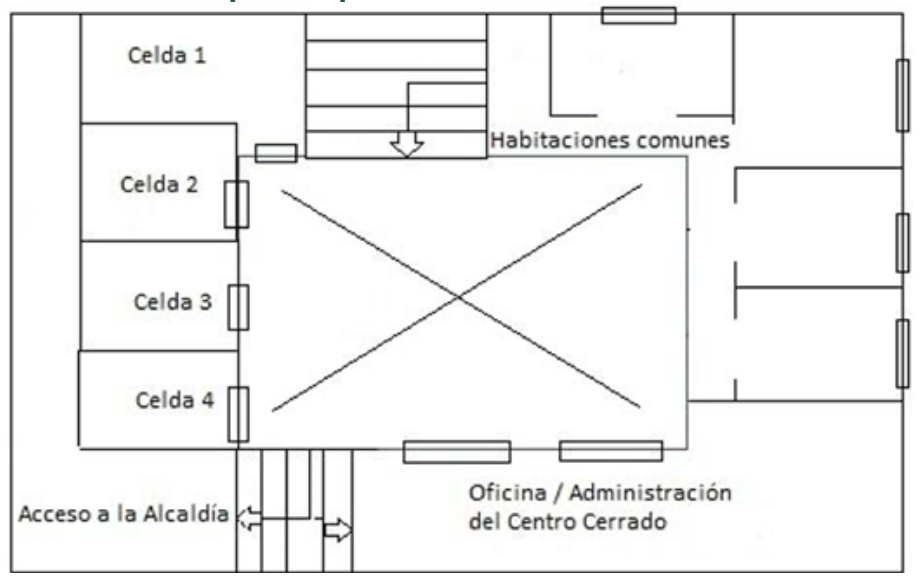

Fuente: elaboración propia.

Como se señaló, la alcaldía constituye un ámbito de ingreso para los jóvenes, es decir, donde se transita la primera etapa de detención en el dispositivo de custodia. Se trata de una instancia de "ablande" y "neutralización" (Daroqui et al., 2012), y de mayor segregación que los jóvenes ubicados en el centro cerrado. Está ubicada en el mismo espacio constructivo, en el piso superior y en el lateral izquierdo, y se exhibe como un espacio significativamente más cruento y de "castigo dentro del castigo" (Daroqui et al., 2012). 
Caracterizar la alcaldía resulta una tarea necesaria para dimensionar las condiciones de degradación, escasez y "reafirmación del poder de castigar" (Daroqui, 2014) que se producen con mayor intensidad en este sector del dispositivo carcelario. En primer término, cabe apuntar que dichas alcaldías no constan dentro de la taxonomía de la Resolución 171 de 2007 que establece la nomenclatura de los dispositivos del Sistema de Responsabilidad Penal Juvenil, como se destacó en la introducción. Tal circunstancia, que agita la imagen de la cárcel como "agujero negro" formulada por Angela Davis $(2003)^{6}$, destaca el marco de ilegalidad en el que estas se despliegan, más allá de que los rasgos que suelen presentar permiten describirlas como espacios de aprehensión y depósito de jóvenes, que generalmente se constituyen como una primera fase de encarcelamiento hasta la derivación hacia uno de los dispositivos previstos en la resolución mencionada. Se señala que son espacios de rezago, cuya "denominación, modalidad y usos asociados son producto de la mera creatividad de las instituciones a la hora de resolver la falta de espacios de encierro", en tanto que los jóvenes "no saben cuánto tiempo estarán allí ni a dónde serán derivados, esto es, la alcaldía como no-lugar es centralmente un tiempo de incertidumbre" (López, 2012, p. 188). Así, en relación con este último punto en particular, el $60 \%$ de los jóvenes entrevistados manifestaron haber sido alojados en la alcaldía entre seis y ocho semanas, un $30 \%$ entre tres y cinco semanas, y solo un $10 \%$ entre una y dos semanas.

Es importante en este punto profundizar en torno a lo que implica la experiencia de encierro en la alcaldía ubicada en el piso superior del centro cerrado. En ella se da, como se ha dicho, lo que en el sistema minoril se conoce como el "ablande", término que designa al estadio primario que atraviesa todo joven institucionalizado, cuando como primera medida se lo deriva a un sector de aislamiento casi total o compartido con otros pocos pares.

El ablande suele tener lugar en superficies muy limitadas, de unos seis metros cuadrados, desprovistas de espacios diferenciados de recreación y en las

6 Según Davis, el capitalismo contemporáneo va de la mano de un creciente y masivo encarcelamiento, y en ese sentido es que la metáfora del "agujero negro" pretendería expresar cierto alineamiento entre las políticas securitarias y los cambios en la morfología del control social a partir de la era posfordista. cuales se genera un ambiente sofocante. La medida se recrudece con una incomunicación absoluta durante varias horas, en las que también se dosifica tanto la exigua provisión de alimentos como las oportunidades de aseo personal. Se trata de una instancia que, en los hechos, neutraliza al joven psíquica y físicamente con miras a su adecuación al régimen del encierro, al tiempo que funciona como antesala a su ubicación con los demás internos. Así, el espacio de la alcaldía se impone como una etapa en la cual el joven es inmerso en las reglas del espacio carcelario, confrontándose asimismo con su propia soledad, y es también "observado" y de algún modo analizado por los operadores judiciales en cuanto a su "perfil" y comportamiento, para luego ser derivado. De este modo, la alcaldía constituye un espacio de quiebre con el afuera (ablande) y de observación institucional para el adentro (clasificación y derivación de los jóvenes).

La alcaldía de la institución de encierro analizada consta de una estructura de cuatro celdas de unos seis metros cuadrados aproximadamente, y cada una contiene en su interior cuatro camastros. Las celdas de máxima seguridad tienen unas ventanas enrejadas de unos $50 \times 50 \mathrm{~cm}$ con escasa vista al patio interno de la institución. De acuerdo con los testimonios de los jóvenes entrevistados que estuvieron alojados en la alcaldía, tanto el frío como el calor se perciben exacerbados en tal ambiente. Todos estos jóvenes manifiestan que la alcaldía es un lugar cruento y celebran haber podido salir de allí. No obstante, la salida de la alcaldía está gobernada por designios que los jóvenes no pueden dominar: en principio, es preciso que las autoridades verifiquen que los jóvenes tienen "buena conducta" -es decir que el ablande los haya neutralizado-, para que puedan en consecuencia ser "bajados", esto es, trasladados al piso inferior -el centro cerrado-, o bien a otra institución. De otra parte, dada la situación de superpoblación tanto en la alcaldía como en el centro cerrado, además de la "buena conducta" es necesario que se produzca el egreso de un joven del centro cerrado para que otro pueda "bajar" de la alcaldía y ocupar su lugar. El director del establecimiento señala que "cuando se egresa uno de ellos, baja uno de arriba" (Entrevista 6. 2018, min. 10:00), discurso indirecto que, sin embargo, no puede dejar de evidenciar un mecanismo de gobierno vinculado a "las formas de penalidad y el cálculo de sus costos" (Foucault, 2006, p. 18), en el cual, más allá de la retórica de los beneficios individuales y del "premio" a la conducta, las posibilidades reales 
de los jóvenes están prefiguradas por las escasas posibilidades que brindan las instalaciones.

Como muestran las figuras 1 y 2, el espacio inferior y superior-derecho del centro cerrado es adyacente al de la alcaldía. Salvo por el hecho de que los jóvenes alojados en la alcaldía pueden ser derivados a otra institución que no sea necesariamente la ubicada en el piso inferior, lo que interesa señalar aquí es que es evidente que entre ambos espacios existe una relación estrecha que va más allá de lo edilicio. En este sentido es que toma relevancia la propuesta del presente artículo, en tanto aquí se traduce con claridad que la forma arquitectónica emerge como una dimensión expresiva de la administración carcelaria. En efecto, los jóvenes hablan de la alcaldía como si esta perteneciera a otra circunscripción espacial, y por eso se refieren a ella de manera tan ajena. Por ejemplo, uno de ellos señalaba: "Dicen que este instituto [el centro cerrado] es de los mejores [...] y sí, nosotros estamos bien, nada que ver con otros lugares, con allá arriba [la alcaldía]" (Entrevista 10, 2018, min. 23:00). Así, la alcaldía aparece por fuera del radio institucional, invisibilizándose la cercanía extrema que conlleva que esté situada en la parte superior del mismo edificio.

En concordancia con las entrevistas y la observación participante, pudo identificarse que, lejos de ser un espacio remoto y una etapa del pasado, la experiencia de privación de la libertad en el marco de la alcaldía funciona como una vivencia visceral, significativa, que signa la trayectoria de los jóvenes en las mallas del sistema penal. Puede afirmarse que el pasaje por la alcaldía se apuntala como un hito omnipresente, cuyo recuerdo cruento en los jóvenes incidirá en la valoración positiva de otros espacios de menor rigor dentro del circuito penal, tal como se verá en los siguientes párrafos en los análisis de las entrevistas. Según Moran (2015), se suele argumentar que la geografía carcelaria no consta solamente de una superficie para la vigilancia y el control, sino también de un medio en el que se desarrollan prácticas y trayectorias humanas, perspectiva útil para analizar espacios como la alcaldía penal, la cual se moldea como un eslabón tan iniciático como determinante en la trayectoria de los jóvenes institucionalizados.

Lo expuesto en el párrafo anterior pudo constatarse una y otra vez durante el trabajo de campo. Desde el patio del centro cerrado, quienes están en el piso inferior pueden alzar la voz y hacer contacto con quienes están alojados en el piso superior de la alcaldía. Sin embargo, se puede observar que, si ambos grupos empiezan a entablar un diálogo, son rápidamente disuadidos por los operadores institucionales: "Si tenés tantas ganas de hablar con ellos [con los jóvenes de la alcaldía], te vamos a subir", puede escucharse en reiteradas oportunidades que uno de ellos les dice jocosamente a los jóvenes del centro cerrado (nota de campo, 28 de marzo de 2018). Y es que los operadores institucionales pueden remitir a estos últimos nuevamente a la alcaldía, por un tiempo que usualmente va de 24 a 48 horas, como forma de castigo adicional ante la comisión de una falta o episodio de quebranto del orden. Es clara así la subsidiariedad entre ambos espacios y la manera como el espacio constructivo entreteje modos diferenciales de ser habitado.

La modalidad de la alcaldía como espacio de castigo y aislamiento impone la posibilidad de pensarla como ámbito de "grado cero penitenciario" (Motto, 2012), es decir, supone un ámbito de total despojo y carencia, donde el rigor y la desposesión se exhiben con toda su crudeza. Los testimonios de los jóvenes también evidencian el contraste entre lo que implica estar situado en uno u otro lugar:

Nada que ver acá [el piso inferior] con allá arriba [por la alcaldía] [...]. Allá es un garrón ${ }^{7}$, cuando es invierno te cagas de frío, cuando es verano te cagás de calor. (Entrevista 10, 2018, min. 20:00)

Acá estamos bien [...]. Allá [en la alcaldía] es un bajón ${ }^{8}$; te levantan a las ocho y media [de la mañana] y ahí ya quedás engomado ${ }^{9}$ hasta las seis de la tarde o hasta la noche. (Entrevista 13, 2018, min. 13:00)

De las respuestas de los jóvenes entrevistados se infiere que siempre están comparando el centro cerrado con la alcaldía y del análisis de los registros de campo puede decirse que conciben y experimentan el espacio físico del dispositivo penal según una economía de usos y prácticas que a su vez delimitan

7 Adjetivación que equivale a infortunio, mal momento o desgracia.

8 Adjetivación que funciona para referenciar un objeto, persona o situación en términos de insuficiencia o deterioro.

9 El "engome" alude a una práctica institucional que implica el encierro de los internos en sus celdas, generalmente en condiciones de mayor separación y aislamiento y durante una gran cantidad de horas. 
una producción estratégica del espacio carcelario. Esta conceptualización de "espacio estratégico" obedece a que las prácticas sociales que allí se despliegan tienen como resultante la configuración de espacios de privilegio, por un lado, y espacios de privación, por el otro, reeditando el sistema de premios y castigos propio de la institución carcelaria. Los testimonios recabados coinciden con lo señalado al respecto por la bibliografía especializada, esto es, que los jóvenes más dóciles son quienes acceden a lugares de mayor beneficio, en tanto que los más "resistentes" o que implican un escollo o desafío al orden interno carcelario suelen ser conducidos a los espacios de mayor "dureza" para su neutralización ${ }^{10}$.

La última nota de campo transcripta evidencia que los jóvenes no quieren volver a la alcaldía, que caracterizan invariablemente como un espacio donde "te tenés que cagar a trompadas ${ }^{11}$ sí o sí. Estás todo el día engomado, uno [un joven] pasa, y te mira mal, y te cagás a trompadas" (Entrevista 10, 2018, min. 8:00). Ante estos discursos que texturizan y moldean las experiencias en el encierro, es válido volver sobre la concepción de lo carcelario como un "archipiélago" (Moran, 2015; López, 2015), que permite inteligir que la construcción de lo "bueno" y lo "malo" al interior de la cartografía penal juvenil constituye una urdimbre relacional en función de la cual emerge "un orden de gobernabilidad interno del sistema, que requiere administrar diferencialmente jóvenes y transformar el acceso a un encierro digno en un beneficio, aun cuando ello implique sostener niveles de sobrepoblación que podrían atenuarse" (López, 2010, p. 18).

Es necesario resaltar, como un asunto significativo en este análisis, que las actividades realizadas por el equipo de extensión universitaria únicamente pudieron desarrollarse en la planta inferior de la institución y los destinatarios fueron solo los jóvenes alojados en las "habitaciones" del lateral derecho del piso superior. Es decir, los jóvenes acuartelados

10 Esta economía del castigo recuerda lo señalado por Walker (2016) a partir de un extenso trabajo de campo realizado en una cárcel del estado de California, donde detectó que la interacción entre los mandatos institucionales y las relaciones sociales producidas en el espacio penal tendían a incrementar los episodios de violencia antes que a reducirlos. Ello reafirma la idea foucaulteana de la cárcel como factoría delictiva.

11 En el habla coloquial, "trompada" puede designar un golpe fuerte accidental o una golpiza. "Cagar a trompadas" equivale, entonces, al desarrollo de una agresión física entre dos o más personas. en la alcaldía no tuvieron acceso a ellas, lo cual reafirma el carácter de dureza y castigo de este espacio, que va más allá de la forma arquitectónica del centro cerrado y crea una idea de "beneficiados" en quienes están fuera de ella.

Este cercenamiento castigado/beneficiado o "lógica del beneficio" plantea que el tránsito hacia una actividad recreativa se reserva a "aquellos presos que exhiben signos claros de obediencia, presos 'que hacen conducta'" (Andersen, 2014, p. 275).

Según lo anterior, cabe preguntarse de qué manera el espacio del centro cerrado trasciende los objetivos de las normas en cuestión, y cómo ello se complejiza a la luz de una economía de prácticas, tránsitos y usos posibles que moldean el espacio arquitectónico en favor de las necesidades e intenciones del gobierno interno institucional.

\section{Prácticas tácticas en el espacio carcelario: fotografía y circulación}

En esta sección se propone focalizar en torno a algunas escenas suscitadas en el marco de los encuentros de extensión que de alguna forma inquietaron la regimentación del ordenamiento interno institucional. El vínculo con el objeto de este artículo está dado por el hecho de que la lógica misma del centro cerrado plantea que la "clientela"12 alojada debe ser despojada de su "cultura de presentación", lo que se configura como una operación de ruptura con su pasado en el mundo exterior y constituye el primer ejercicio de una larga cadena de actos en la dirección de "mutilar el yo" (Goffman, 1961).

En primer lugar se destaca un encuentro realizado en la segunda mitad del 2018, para el cual se distribuyeron cámaras fotográficas. Los jóvenes se sumaron a tomarse fotos, juntos y separados, sonriendo, abrazándose. Alan, uno de ellos, le pidió a una extensionista que le tomara una foto sonriendo y al terminar la jornada le preguntó si sería posible imprimirla para regalársela a su madre.

12 La idea de "clientela" pretende dar cuenta de la selectividad del sistema penal, en la medida en que son determinadas capas sociales las que mayoritariamente se constituyen como objeto de persecución penal, políticas de encarcelamiento y otras estrategias complementarias de gobierno. Desde la perspectiva criminológica, podría señalarse que el concepto se asocia a las teorías del etiquetamiento (labeling approach), que enfatizan cómo se despliegan los procesos de criminalización. 
La docente accedió y se la entregó en el siguiente encuentro. Dos semanas más tarde, sin embargo, esto aconteció:

Lo veo a Alan y le pregunto qué le pareció la foto que una de las docentes le había impreso, a lo que él esboza una sonrisa irónica. Afirma, algo sorprendido por la pregunta, "Decile a ella que te cuente [señalando a una docente] [...]. Decile a ella que te cuente". Minutos más tarde, cuando le pregunto a la docente en torno al tema, ella indica que Alan le contó que parecía ser que, en una de las requisas, los "maestros" habían encontrado la fotografía, lo que motivó que el director le diera un escarmiento al joven. Al mismo tiempo, la docente expresa que va a llamar al director para ver cuál fue el "malentendido". (Nota de campo, agosto de 2018)

Como se observa en la nota, se atropelló la intimidad del joven Alan al arrebatarle la fotografía que iba a darle a su madre cuando lo visitara. La escena evidencia claramente la crueldad del castigo, pues más allá de lo que pudiera prescribir el reglamento en torno a las pertenencias personales, es evidente la falta de proporcionalidad entre el hecho en sí y la represión efectuada por el directivo. Asimismo, toma forma aquí la operatoria propia de la institución total, en la medida en que "la falta de gavetas individuales, así como los registros y las confiscaciones periódicas de objetos personales acumulados, refuerzan el sentimiento de desposesión" (Goffman, 1961, p. 31). En ese sentido, el decomiso de la fotografía es otra práctica encolumnada en el orden de la "desintegración del yo".

La escena de disputa en soledad entre un joven y los operadores institucionales ("maestros", director) ejemplifica lo que se ha denominado "régimen despótico de la prisión" (Chauvenet, 2006), cuyo elemento central es la violencia institucional. No obstante, es menester apuntar que la petición de Alan constituye una práctica de resistencia contra las pautas institucionales, y el taller extensionista se concibe como un facilitador para la consecución de su objetivo.

En efecto, y siguiendo la línea argumental de lo apuntado en el párrafo anterior, debe señalarse que la fotografía en cuestión capturaba una imagen del joven sonriendo, exhibiendo felicidad, lo que significa muchos aspectos disruptivos para el orden interno del establecimiento. En primer lugar, la fotografía daba cuenta de la circulación de una imagen de un joven en el mundo exterior al centro cerrado. Era evidente que las extensionistas habían tomado esa fotografía, la habían imprimido y finalmente se la habían dado a Alan para su apoderamiento o para que él se la entregara a su madre. Ciertamente, el hecho había quebrado la divisoria entre el "adentro" y el "mundo exterior" propio de la "institución total". Este gesto recuerda que "la expresión cultural es, ante todo, una operación" (De Certeau, 2000, p. 200), pues la imagen de Alan capturaba un momento dado de su existencia, una trayectoria a contrapelo de los modos de circulación habilitados y prescriptos por la institución penal, eminentemente anclados en un régimen de incomunicación con el exterior.

En términos de Sontag (2014), la fotografía es una forma de representación que, al igual que la pintura, capta la realidad y la interpreta, lo que desecha toda idea de objetividad. Sin embargo, la particularidad del dispositivo fotográfico reside en que no se trata solo de una interpretación de la realidad, sino que es también un vestigio material, un rastro directo de lo real. En este caso, la fotografía de Alan era un rastro de su autopercepción y el testimonio de una ayuda externa que le había facilitado la materialización de esta operación subalterna. Desde este punto de vista, tiene coherencia que la imagen en cuestión haya sido eliminada; ni enviada a su familia ni devuelta a las docentes, sino eliminada.

La posibilidad de concebir la práctica de Alan como táctica adquiere sentido si se la analiza en relación con el espacio en el cual tuvo lugar. Tal como señala Goffman (1961, p. 21), "en las instituciones totales hay una escisión básica entre un gran grupo manejado adecuadamente conformado por los internos, y un pequeño grupo personal supervisor". Tomando como punto de partida lo anterior, cabe señalar que en múltiples partes del centro cerrado pueden encontrarse cámaras de seguridad cuyo objetivo es constatar y registrar la actividad de los actores que lo transitan. Ahora bien, esta presencia de las cámaras divide el establecimiento en dos partes en contraste: la de los observadores y la de los observados. Así pues, esta habilitación entre quienes son los que observan y tienen la potestad de registrar y capturar una situación mediante la imagen y quienes no fue justamente la que se puso en entredicho.

Para Foucault (1995), la resistencia es tan dispersa como el poder, es decir, es "tan inventiva, tan móvil, tan productiva como él. Es preciso que, como 
el poder, se organice, se coagule y se cimiente. Que vaya de abajo arriba, como él, y se distribuya estratégicamente" (p. 162). De esta manera, resulta interesante pensar la producción de una resistencia en función de la fotografía de Alan, pues más allá de la reprensión efectuada por el director que luego devino en una reorganización del escenario de poder, por un momento puso en entredicho la validez de la división instituida entre observadores y observados. Retomando las palabras de Sontag (2004), la fotografía era un vestigio material de la suspensión o interrupción de ese orden carcelario.

En este punto es válido traer a colación un comentario de Sykes (2017), quien conceptualiza la administración del espacio en las instituciones penales norteamericanas. Tras las prácticas securitarias que allí observó, dedujo que el ideal de securitización se develó como un fin en sí mismo, antes que como un medio. El efecto de esta circunstancia, según este autor, es que "las interminables precauciones, el recuente constante de la población de internos, la miríada de regulaciones, la sospecha institucionalizada de las requisas periódicas son la expresión de medios injustificados y de odio antes que de razón" (Sykes, 2017, p. 67). En consonancia con esta interpretación -vinculada al mencionado "régimen despótico de la prisión"-, puede aseverarse que el espacio debe ser constantemente vigilado y semantizado en términos de la gobernabilidad institucional, de manera que las ficciones creadas y difundidas en torno a este (sobre la resocialización, el respeto a los derechos de los jóvenes, el castigo terapéutico, entre otras) pueden ser muy rápidamente puestas en entredicho ante acontecimientos contingentes que delatan la arbitrariedad de la institución penal y el predominio de sus necesidades de orden interno sobre cualquier otro elemento. Así, y pasando del esquema de poder de Foucault $(2005 ; 1994)$ para focalizar la escena de la fotografía desde las contribuciones de De Certeau (2000), puede argumentarse en consecuencia que la operación efectuada por el joven significó una "táctica" débil y silenciosa, es decir, una de aquellas producciones que "se forman e identifican gracias a la posibilidad de situarse en alguna parte en relación con las otras fuerzas" ( $p$. 169), que generó una marca efímera pero sensible en el espacio penal, sobre todo si se la mide en relación con la enorme reprimenda que motivó.

Lo expuesto con relación a la fotografía de Alan marca la pauta del tipo de prácticas simbólicas que podían suscitarse al tenor de las experiencias extensionistas. El encuentro de extensión tuvo tal plasticidad o apertura o desestructuración, que funcionó como una plataforma donde el joven pudo enlazar su práctica -ciertamente disruptiva-, posicionando un uso del espacio alternativo al dado y gerenciado por la institución. Esto tiene directa vinculación con el objetivo de este artículo, en la medida en que el ingreso de la lógica educativa permitió en este caso ser un sustento para las necesidades propias de uno de los jóvenes, que promovió la expresión individual en un espacio fuertemente disciplinado. Incluso, el hecho de que el director haya reprendido al joven, pero no le haya dicho absolutamente nada al respecto a las docentes del taller, evidencia la discreción con la que se pretendió recubrir el acto de violencia institucional sobre Alan. En este punto puede argumentarse que, por lo observado en el campo, el director del establecimiento priorizó no confrontar directamente con las extensionistas, lo que de alguna manera le permitió a él sostener un ideario también pedagógico a los ojos de ellas. Lo contrario habría implicado que el representante institucional desnudara la centralidad del mandato de seguridad, circunstancia constantemente disimulada en el ideario de las instituciones de encierro juvenil.

En línea con lo dicho hasta aquí, cabe mencionar otras escenas en las que se concibe nuevamente el taller de extensión universitaria como canalizador de prácticas tácticas de los jóvenes en relación con el espacio habitado. En una ocasión, dos de las docentes alentaron a Federico, uno de los reclusos, a cantar una canción que había escrito, ya que este también hacía rap ${ }^{13}$, a lo cual el joven accedió:

Ya no soy el mismo que era ayer / Son tantas las cosas que hoy puedo entender / el tiempo también nos cambia la mirada / el tiempo va pasando y aquí yo sigo estando / extrañando y recordando lo que yo hacía a diario / son tantos los recuerdos que ocupan en mi mente / que me dan ganas de volver el tiempo y hacer las cosas diferente / [...] Pronta libertad. (Registro de campo, 14 de septiembre de 2018)

Las docentes filmaron el performance en el SUM del centro cerrado, registrando también al resto de jóvenes, que habían prestado mucha atención a la

13 Estilo musical de origen afroamericano en que, con un ritmo sincopado, la letra, de carácter provocador, es más recitada que cantada. 
creación de su compañero. Al término de la actividad, Federico quiso comprometer a las docentes a hacerle llegar el video a su madre. Como este episodio fue posterior a lo ocurrido con Alan, ellas optaron por consultar si podían acceder al pedido, lo que fue rechazado por los operadores de la institución, con el argumento de que estaba prohibido que circularan imágenes de los jóvenes privados de la libertad y de jóvenes menores de 18 años.

Lo que interesa señalar como corolario de esta escena es su vinculación con la experiencia extensiva, en la medida en que esta también podía posibilitarle la concreción de un objetivo preciso: que su familia supiera que estaba componiendo y cantando en la cárcel. Esta circunstancia traduce nuevamente cierta resistencia, que se expresa en términos de táctica. Como en parte se ha explicado, las tácticas son efectuadas por practicantes sin un "lugar propio", que en consecuencia se verán marcadas por la inestabilidad de su trazo. De Certeau (2000) señala que "hay que volverse de cara a estas prácticas, a aquella proliferación diseminada de creaciones anónimas y perecederas, que no se capitalizan pero hacen vivir" (p. 18). De conformidad con la última parte de la cita, puede argumentarse que la práctica táctica de Federico no pudo capitalizarse de lleno, en tanto no consiguió el objetivo de hacerle llegar el video a su madre. Sin embargo, el hecho de que su pedido haya atravesado el espacio de extensión universitaria para canalizarse como una solicitud formal a las docentes demuestra que los jóvenes están lejos de la inercia y la completa neutralización. En la operación de Federico se evidenció nuevamente el ímpetu por desafiar la traza espacial que separa el adentro del afuera. Otra prisión más allá de los muros es la que gobierna esta desconexión de las familias respecto de lo que sucede con sus hijos, tal como lo expone Chauvenet $(1998 ; 2006)$ en una investigación sobre esta temática en particular.

La misma situación se presentó con frecuencia durante otros encuentros en los cuales se trabajó con cámaras fotográficas o videofilmadoras. Los jóvenes se entusiasmaban con tomar capturas haciendo muecas o exhibiéndose en grupos de amigos, abrazándose o saludándose. Posteriormente, surgía en ellos la voluntad de que sus familias tuvieran conocimiento de estos momentos de juego y creatividad, lo cual era imposible a la luz de las restricciones y recaudos que, incluso en nombre de la seguridad de los propios jóvenes, eran enarboladas por los operadores institucionales. El resultado permite analizar no solo cierta situación de frustración propulsada por la lógica de gobierno, sino la imposibilidad de que el espacio penal fuera traspuesto. Cabe señalar que las familias de los jóvenes eran ajenas a lo que sucedía con ellos en el marco de las experiencias de taller, lo que se constituye como una incomunicación propia de la "experiencia carcelaria de la privación" reseñada por Touraut (2012).

Las dos escenas analizadas permiten conectar la experiencia de extensión universitaria con las prácticas tácticas de los jóvenes. Esto resulta muy significativo teniendo en cuenta los propósitos analíticos de este artículo, en la medida en que se evidencia cómo lo producido en el marco del taller, o en ocasión de este, es reservado para la vida intra muros, anulando la institución penal la posibilidad de que las prácticas mencionadas emerjan o trasciendan hacia otros espacios, como el exterior, que involucra a las familias de los jóvenes. Esta constatación implica una limitante para las escenas pedagógicas, en la medida en que la divisoria institución/mundo exterior impide que una escena educativa rebase el espacio de punición ${ }^{14}$.

\section{CONCLUSIONES}

Tal como se señaló en el marco teórico, la cuestión de la organización del espacio constituye una dimensión productiva para analizar el despliegue de prácticas de poder en un dispositivo punitivo como lo es la prisión. Ya en el clásico Vigilar y castigar (2005), Michel Foucault advirtió con suspicacia que, más allá de las mutaciones significativas que atravesó la administración del castigo penal en los últimos siglos, cierto es que este nunca dejó de ser corporal. Esta mirada, que permite pensar en la continuidad del abordaje conductual de la "clientela" penal, evidencia que la distribución de los internos, la organización de espacios de reprensión y la mecánica de gobierno que autoriza los tránsitos posibles en el encierro constituyen claves de análisis productivas para el abordaje del "despliegue real del castigo" (Daroqui, 2014).

Desde la perspectiva señalada, el análisis cualitativo de los datos construidos a partir de la experiencia de

14 En otras oportunidades (Urtubey, 2020) se han realizado indagaciones con mayor profundidad en torno a las tensiones suscitadas con ocasión del despliegue de experiencias educativas, en dispositivos de encierro punitivo de jóvenes. 
campo denota que la implantación de la alcaldía en un espacio de la planta superior del centro cerrado y sus formas de circulación constituye ciertamente una modalidad típica de segmentación del poder punitivo al interior de las geografías carcelarias (Moran, 2015; Motto, 2012). Las prácticas y dinámicas institucionales que rodean a la alcaldía ponen de manifiesto un caso de producción de espacios diferenciales al interior de la institución penal. El análisis de las entrevistas realizadas a los jóvenes, asimismo, destacó que la diferenciación del espacio está dada por una aplicación de pura agresión y de "suplemento punitivo" (Foucault, 2005).

Finalmente, debe recordarse que, tal como se ha señalado desde la geografía constructivista, toda sociedad produce constantemente espacios donde se "permite que tengan lugar determinadas acciones, sugiere unas y prohíbe otras" (Lefebvre, 2008, p. 129). Los resultados colectados en la investigación base de este artículo dan cuenta en el estudio de caso que la forma arquitectónica de un establecimiento penal es clave en la producción estratégica del espacio carcelario. Así, el análisis de la alcaldía arrojó que su implantación en el espacio contribuyó a sedimentar y establecer ámbitos de una mayor "dureza", por un lado, y espacios más blandos o "de beneficio", por el otro. En efecto, la imposibilidad de acceso del equipo de extensión universitaria a dicho espacio da cuenta de esta geografía compleja y eminentemente práctica, absolutamente indiferente a las discursividades jurídico-dogmáticas sobre la infancia y la juventud en conflicto con la ley penal. 


\section{REFERENCIAS}

Andersen, M. J. (2014). La penalidad neoliberal en el siglo XXI: la tercerización del gobierno carcelario a través de la "gestión evangelista penitenciaria" en las cárceles bonaerenses. Tesis de Maestría, Universidad de Mar del Plata-Universitat de Barcelona, Buenos Aires, Argentina.

Bourdieu, P. (1997). Razones prácticas. Sobre la teoría de la acción. Barcelona: Anagrama.

Bourdieu, P. (1999). La miseria del mundo. Buenos Aires: Fondo de Cultura Económica.

Daroqui, A. (2014). Castigar y gobernar. Hacia una sociología de la cárcel. La Plata: Comisión Provincial por la Memoria-Grupo de Estudios sobre Sistema Penal y Derechos Humanos.

Daroqui, A., López, A. L. \& Cipriano, R. (2012). Sujeto de castigos. Hacia una sociología de la penalidad juvenil. Rosario: Homo Sapiens.

Davis, A. (2003). Are Prisons Obsolet? Nueva York: Seven Stories Press.

De Certeau, M. (2000). La invención de lo cotidiano. México: iteso.

Foucault, M. (1995). El sujeto y el poder. En O. Terán (Comp.). Michel Foucault. Discurso, poder y subjetividad. Buenos Aires: El Cielo por Asalto.

Foucault, M. (2005). Vigilar y castigar. El nacimiento de la prisión. Buenos Aires: Siglo XXI.

Foucault, M. (2006). Seguridad, territorio, población: Curso en el Collège de France 1977-1978. Buenos Aires: Fondo de Cultura Económica.

Goffman, E. (1961). Internados. Ensayos sobre la situación social de los enfermos mentales. Buenos Aires: Amorrortu.

Guemureman, S. (Comp.). (2015). Políticas penales y de seguridad dirigidas hacia adolescentes y jóvenes. Santa Fe: Rubinzal Culzoni.
Lefebvre, H. (1991). The Production of Space. Oxford: Blackwell.

Lefebvre, H. (2008). Espaço e política. Belo Horizonte: Editora UFMG.

López, A. L. (2010). Proceso de reforma legal e institucional del sistema penal juvenil en la Provincia de Buenos Aires (2000-2009). Tesis de Maestría Universidad de Buenos Aires, Buenos Aires, Argentina.

López, A. L. (2012). Cuerpo y subjetividad en el gobierno institucional del encierro juvenil. Sociedad y Equidad, 3, 45-65.

López, A. L. (2015). Prácticas y alcances del sistema penal juvenil en la provincia de Buenos Aires. Ponencia presentada en las I Jornadas de Sociología de la Facultad de Ciencias Políticas y Sociales de la Universidad Nacional de Cuyo, Mendoza, Argentina.

Moran, D. (2015). Carceral Geography: Spaces and Practices of Incarceration. Farnham: Ashgate.

Motto, C. (2012). Los usos de la violencia en el gobierno penitenciario de los espacios carcelarios. Question, 36(1), 69-80.

Scribano, A. (2008). El proceso de investigación social cualitativo. Buenos Aires: Prometeo.

Urtubey, F. E. (2020). Extensión universitaria con jóvenes en conflicto con la ley penal. Análisis de experiencias en dos dispositivos de encierro punitivo del Sistema de Responsabilidad Penal Juvenil de la provincia de Buenos Aires, Argentina (2018-2019). Tesis doctoral Universidad Nacional de La Plata, Buenos Aires, Argentina.

Valles, M. (2000). Técnicas cualitativas de investigación social. Madrid: Síntesis.

Walker, M. L. (2016). Race Making in a Penal Institution. American Journal of Sociology, 121(4), 1051-1078. 